\title{
GENERAL PRINCIPLES FOR THE CONSTRUCTION OF ORDINAL SCALE ${ }^{1}$ \\ 1. PRINCIPLES OF FREQUENGY DISTRIBUTION
}

\author{
YOSHIHISA TANAKA \\ University of Tokyo
}

\begin{abstract}
Three major principles for the ordinal scaling are suggested. The first one based on the number of choice is called the principle of frequency distribution. The application of the principle to the pick one method and the category method is discussed. Possible distortion of order owing to the imperfect reliability of the method or the subjects is analysed.
\end{abstract}

Treatment of data in terms of the ordinal scale is a sound way for the analysis of psychological data since most of psychological events can safely be measured in terms of the ordinal scale. But as the amount of information contained in the ordinal scale is rather small and as the applicability of the mathematical treatment is limited, many attempts have been made to transform ordinal data into ratio or interval ones. On the other hand, it should be pointed out that there is always some danger in the transformations as most of them depend on some assumptions, the validity of which is hardly verified. If some of the basic assumptions underlying the transformation do not hold for the process under consideration, the treatment of data in terms of ratio or interval scale is quite misleading.

The basic assumptions for an ordinal scaling are simpler and more straightforward than those of interval or ratio scaling, but the rationale underlying the ordinal scaling is not always so simple and straightforward as it seems at the first glance. Therefore, it is necessary for us to consider the major principles under which ordinal scales are constructed. It is also necessary for us to consider the major types of models

1 This paper is a part of research supported by a Grand in Aid for Scientific Research, Ministry of Education. underlying psychometrics in order to clarify these principles.

As we have already discussed the details of these models (Tanaka, 1966), we will repeat only the main points Two types of models are proposed; the deterministic model and the variability model. The first type takes the assumptions that (1) the relationships between stimulus variables (S-variables), intervening psychological variables ( $\mathrm{P}$-variables) and response variables (R-variables) are definite and stable, and that (2) there is no variability within these variables. Most of the psychological models have deterministic aspects and we can conclude that most of the variability models have something in common with the deterministic models.

Four types of variability models are proposed ; S-P variability model, P-variability model, S-P: $\mathrm{P}$ variability model and P-R variability model. The first one assumes the existence of variability in the relationship between $\mathrm{S}$ - and $\mathrm{P}$ - variables, the second one assumes the variability in $\mathrm{P}$ variables, the third one is the combination of the first and the second ones and assumes variabilities not only in the S-P relationship but also within $\mathrm{P}$-variables, and the last one assumes variability in the $\mathrm{P}-\mathrm{R}$ relationship.

It is possible to classify the major principles of the ordinal scaling into three types according to the nature of obtained data 
as well as the basic assumptions for the treatment of the data. We have already suggested that a set of $n$ objects can be ordered according to the frequency at which each one of them was chosen by $S$ s. Let us call this type the principle of frequency distribution. In the second major type, a set of stimuli or items is ordered according to the results obtained by refering to a known scale. It is also possible to decide an order of a set of objects or events according to the results obtained by the mutual comparisons among them. Let us call this type the principle of mutual comparisons. Sometimes, an ordinal scale is constructed according to one of these principles but it sometimes happens that a combination of these principles is used for the construction of an ordinal scale. We will primarily be concerned with the principle of frequency distribution in the present paper.

\section{The principle of frequency distribution}

It sometimes happens that the order of objects is determined by counting the number of choice. Many decisions are made on the basis of the number of votes. A girl who earns the largest number of support receives the first prize and the one who earns the next largest number of votes receives the second prize and so forth. These instances clearly indicate that frequency distribution is sometimes of grave importance in social life.

A typical case for the principle of frequency distribution is as follows: Elements of a set of stimuli are presented to a subject one by one and the subject is requested to sort the stimuli into different categories according to a certain criterion. The nature of the categories is not necessary ordinal. One of the simplest instances of the sorting may be that of A and non-A type. A subject may be requested to repeat the sorting or a group of subjects is used for the sorting.

Another type of the application of the principle can be found in the pick $m$ method where subject is requested to pick $m$ objects out of $n$ according to a certain criterion. The simplest case of the method is the pick one. For example, the stimuli used are several statements concerning a political issue and a subject is requested to choose one with which he agrees. He is requested to make the choice in several occasions. Let us imagine what will happen in the case when a deterministic model is applicable. The meanings of the statements are so clear that there is no possibility that the $\mathrm{S}-\mathrm{P}$ relation fluctuates. The attitude of the subject toward the political issues is so definite that he always chooses the same statement. The obtained data only indicate that the subject chooses the same statement. The same thing may happen when the procedure is applied to a group of subjects whose attitudes toward the political issue coincide with each other. The same thing may happen in the case of sorting. We can not apply the principle for these cases. Let us, then, consider the case of variability models.

\section{Pick one method}

Let us imagine that subjects' attitudes toward the political issue differ from person to person although a deterministic model is applicable to each member of the group subjects. Number of selections for the items differs owing to the difference of the attitudes of the subjects. If the statement $\mathrm{A}$ is chosen by $60 \%$ of the subjects, B $30 \%$ and $\mathrm{C}$ is chosen only by $10 \%$ of the subjects, we can decide the order of the three statements according to the number of selections. But this can be done under the assumption that all of the selections have equal weight. If the weight differs from person to person, we should take the factor into consideration. We will restrict our concern to the equal weight situation. The model underlying the analysis is a kind of P-variability model.

Let us next discuss the other cases of variability models. We have already pointed out that frequency distribution can be 
distorted according to the variability or reliability of the subjects or the methods (Tanaka, 1966). Only the cardinal points of the discussion will be repeated here.

Let $f_{1} f_{2} f_{3} \ldots f_{n}$ be the relative frequencies of selection for $n$ items which can be obtained by the pick one method under an ideal situation. The row of frequencies can be shown as row vector $A$. Let $f^{\prime}{ }_{1} f^{\prime}{ }_{2}$ $f^{\prime}{ }_{3} \ldots f^{\prime}{ }_{n}$ be the obtained frequencies which can be shown as row vector $B$. And let $W$ be the reliability matrix of the subjects or the method. $W$ should be an identity matrix when the reliability is perfect. There are many causes which reduce the reliability. S-P variability is the one and the P-R variability is another. In some cases, P-variability enters into the picture. We will discuss the effects of reduced reliability disregarding the possible sources of disturbances.

The relationship between $A, W$ and $B$ can be shown

$$
B=A \cdot W
$$

If the reliability is perfect, $W$ is an identity matrix. Then the relation is

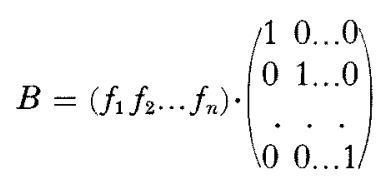

It takes the general form,

$$
B=\left(f_{1} f_{2} \ldots f_{n}\right) \cdot\left(\begin{array}{c}
W_{11} W_{12} \ldots W_{1 n} \\
W_{21} W_{22} \ldots W_{2 n} \\
\ldots \ldots \ldots \ldots \\
W_{n 1} W_{n 2} \ldots W_{n n}
\end{array}\right)
$$

There is one important difference between the present problem and the former one. In the present context of the discussion, the distortion of the frequencies is not a serious problem as far as the order of the items based on the frequency distribution remains the same as the original one. If we take the equation (3) at its face value, anything can happen. But we need not discuss all of the possibilities because we can assume that most of the research or experiments well designed and well performed may nearly accomplish their purposes and may obtain the results which may not be far from the original ones. Therefore, what we should discuss here is the effects of relatively small distortions in the reliability matrix.

Let us first discuss the simplest case where $n=2$. Then the equation (2) can be written

$$
\begin{aligned}
B & =\left(f_{1} f_{2}\right) \cdot\left(\begin{array}{ll}
W_{11} & W_{12} \\
W_{21} & W_{22}
\end{array}\right) \\
& =\left(f_{1} W_{11}+f_{2} W_{21} f_{1} W_{12}+f_{2} W_{22}\right)
\end{aligned}
$$

where $f_{1}+f_{2}=1, W_{11}+W_{12}=W_{21}+W_{22}=1$ We assume that $f_{1}>f_{2}, W_{11}>W_{12}$ and $W_{22}>W_{21}$. We expect that $f^{\prime}{ }_{1}>f^{\prime}{ }_{2}$.

Therefore,

$$
\begin{aligned}
& f_{1} W_{11}+f_{2} W_{21}-f_{1} W_{12}-f_{2} W_{22} \\
= & f_{1}\left(W_{11}-W_{12}\right)-f_{2}\left(W_{22}-W_{21}\right)>0
\end{aligned}
$$

It is obvious that (5) holds under the condition

$$
\left(W_{11}-W_{12}\right) /\left(W_{22}-W_{21}\right)>f_{2} / f_{1}
$$

The following example will clearly show what will happen when (6) does not hold. If the item A gets $60 \%$ support and the item B gets $40 \%$ support under an ideal situation where there is no ambiguity in the meaning of the statements and the responses of the subjects are perfectly reliable. Let us assume that the both items are somewhat misleading and only $60 \%$ of those who will really support the item $A$ choose the item. Let us also assume that the item $\mathrm{B}$ is chosen by $70 \%$ of those who will potentially support it. The result can be given by

$$
\begin{array}{r}
\left(\begin{array}{ll}
0.60 & 0.40
\end{array}\right) \cdot\left(\begin{array}{ll}
0.60 & 0.40 \\
0.30 & 0.70
\end{array}\right) \\
=\left(\begin{array}{ll}
0.48 & 0.52
\end{array}\right)
\end{array}
$$

We will reach a false conclusion that $\mathrm{A}<\mathrm{B}$. This kind of things may easily happen in the society where there is always some 
danger in expressing one's own opinion. The same thing may happen when the subjects are biased against the research, or the items contain some misleading phrases.

We can extend the same reasoning to the case where $n=3$. The relation can be written

$$
\begin{aligned}
B= & \left(f_{1} f_{2} f_{3}\right) \cdot\left(\begin{array}{l}
W_{11} W_{12} W_{13} \\
W_{21} W_{22} W_{23} \\
W_{31} W_{32} W_{33}
\end{array}\right) \\
= & \left(f_{1} W_{11}+f_{2} W_{21}+f_{3} W_{31} f_{1} W_{12}+f_{2} W_{22}\right. \\
& \left.+f_{3} W_{32} f_{1} W_{13}+f_{2} W_{23}+f_{3} W_{33}\right)
\end{aligned}
$$

where $f_{1}+f_{2}+f_{3}=1, W_{11}+W_{12}+W_{13}=W_{21}$ $+W_{22}+W_{23}=W_{31}+W_{32}+W_{33}=1$

We assume that $f_{1}>f_{2}>f_{3}$ and $W_{11}>W_{12}$ $=W_{13}, W_{22}>W_{21}=W_{23}, W_{33}>W_{31}=W_{32}$. From these assumptions, we expect that

$$
\begin{aligned}
& f_{1} W_{11}+f_{2} W_{21}+f_{3} W_{31}-\left(f_{1} W_{12}+f_{2} W_{22}+f_{3} W_{32}\right)>0 \\
& f_{1} W_{11}+f_{2} W_{21}+f_{3} W_{31} \\
& \quad-\left(f_{1} W_{13}+f_{2} W_{23}+f_{3} W_{33}\right)>0 \\
& f_{1} W_{12}+f_{2} W_{22}+f_{8} W_{32} \\
& \quad-\left(f_{1} W_{13}+f_{2} W_{23}+f_{3} W_{33}\right)>0
\end{aligned}
$$

(9) can be written

$$
\begin{aligned}
& f_{1}\left(W_{11}-W_{12}\right)-f_{2}\left(W_{22}-W_{21}\right) \\
& \quad-f_{3}\left(W_{32}-W_{31}\right)=f_{1}\left(W_{11}-W_{12}\right) \\
& \quad-f_{2}\left(W_{22}-W_{21}\right)>0
\end{aligned}
$$

Therefore, the order of $f^{\prime}{ }_{1}$ and $f^{\prime}{ }_{2}$ coincides with the original one under the condition

$$
\left(W_{11}-W_{12}\right) /\left(W_{22}-W_{21}\right)>f_{2} / f_{1}
$$

We can deduce from (10) that

$$
\left(W_{11}-W_{13}\right) /\left(W_{38}-W_{31}\right)>f_{3} / f_{1}
$$

and from (11)

$$
\left(W_{22}-W_{23}\right) /\left(W_{83}-W_{32}\right)>f_{8} / f_{2}
$$

We can easily generalize the results and conclude that the obtained order based on the frequencies coincides with the original one under the condition

$$
\left(W_{j j}-W_{j k}\right) /\left(W_{k k}-W_{k j}\right)>f_{k} \mid f_{j}
$$

where $f_{j}>f_{k}$ and when we can assume that $W_{j j}>W_{j k}=W_{j m}$ and $W_{k k}>W_{k j}=W_{k m}$

\section{Category method}

The general type of the category method is to sort $n$ stimuli into $m$ categories but we will first deal with the case when $m=2$. For instance, the stimuli are consisted of $n$ items concerning a political issue and the subject is requested to answer whether he agrees each item or not. If the forced choice technique is applied to a group of subjects, we obtain two series of frequency distribution, the frequency distribution of agreement and that of disagreement. If the items are ordered according to the frequencies of agreement, the obtained order coincides with that obtained from the frequencies of disagreement, provided that the former is ordered from the highest to the lowest and the latter from the lowest to the highest. Therefore, it is not necessary to consider the both results, but in order to make the principle of our analysis clear, we will deal with the both series.

In the case of pick one method, the subject is requested to choose one item out of $n$ items according to a certain criterion. But in the case of category method, the subject is requested to choose one category out of $m$ categories for each item. Therefore, the error occurs in selecting the categories. Therefore, the relationship between the ideal selection and obtained one under imperfect reliability can be shown by

$$
\begin{aligned}
& B=\left(\begin{array}{cc}
f_{11} & f_{12} \\
f_{21} & f_{22} \\
\cdot & \cdot \\
f_{n 1} & f_{n 2}
\end{array}\right) \cdot\left(\begin{array}{ll}
W_{11} & W_{12} \\
W_{21} & W_{22}
\end{array}\right) \\
& =\left(\begin{array}{cc}
f^{\prime}{ }_{11} & f^{\prime}{ }_{12} \\
f^{\prime}{ }_{21} & f^{\prime}{ }_{22} \\
\cdot & \cdot \\
f^{\prime}{ }_{n 1} & f^{\prime}{ }_{n 2}
\end{array}\right)
\end{aligned}
$$

where $f_{n 1}$ means the relative frequency of response 1 for the item $n$ and $f_{n 2}$ is the rela- 
tive frequency of response 2 for the item $n$, and $W_{11}$ means the probability that response 1 occurs when that response should occur and $W_{12}$ is the probability that response 2 occurs when response 1 should occur. Therefore, $f_{11}+f_{12}=f_{21}+f_{22}=\ldots$. $=f_{n 1}+f_{n 2}=1$ and $W_{11}+W_{12}=W_{21}+W_{22}$ $=1$. Let $F$ be the original frequency matrix, $W$ be the reliability matrix and $B$ be the obtained one, the relationship can be written

$$
B=F \cdot W
$$

The equation (17) assumes that the same $W$ can be applicable to all of the items. If the assumption holds true, $f^{\prime}{ }_{i 1} f^{\prime}{ }_{i 2}$ and $f^{\prime}{ }_{j 1} f^{\prime}{ }_{j 2}$ can be written as

$$
\begin{aligned}
& \left(\begin{array}{l}
f_{i 1}^{\prime} f_{i 2}^{\prime} \\
f^{\prime}{ }_{j 1} f^{\prime}{ }_{j 2}
\end{array}\right)=\left(\begin{array}{l}
f_{i 1} f_{i 2} \\
f_{j 1} f_{j 2}
\end{array}\right) \cdot\left(\begin{array}{ll}
W_{11} & W_{12} \\
W_{21} & W_{22}
\end{array}\right) \\
= & \left(\begin{array}{l}
f_{i 1} W_{11}+f_{i 2} W_{21} f_{i 1} W_{12}+f_{i 2} W_{22} \\
f_{j 1} W_{11}+f_{j 2} W_{21} f_{j 1} W_{12}+f_{j 2} W_{22}
\end{array}\right)
\end{aligned}
$$

If $f_{i 1}>f_{j 1}$, we expect that

$$
f_{i 1} W_{11}+f_{i 2} W_{21}-\left(f_{j 1} W_{11}+f_{j 2} W_{21}\right)>0
$$

(20) can be written as

$$
W_{11}\left(f_{i 1}-f_{j 1}\right)-W_{21}\left(f_{j 2}-f_{i 2}\right)>0
$$

It is obvious that $f_{i 1}-f_{j 1}=f_{j 2}-f_{i 2}$. Therefore, the obtained order of $i$ and $j$ coincide with the original one, when

$$
W_{11}>W_{21}
$$

When $m$ equals three, the equation (18) can be written

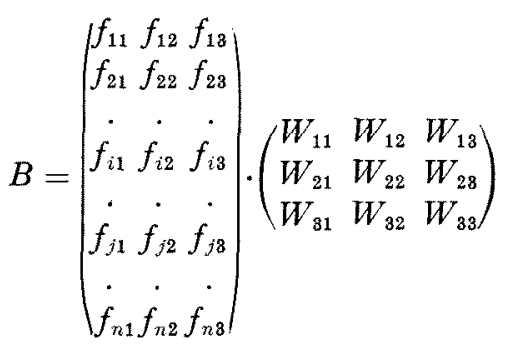

where $f_{11}+f_{12}+f_{13}=f_{i 1}+f_{i 2}+f_{i 3}=f_{j 1}+f_{j 2}$ $+f_{i 3}=f_{n 1}+f_{n 2}+f_{n_{3}}=1, W_{11}+W_{12}+W_{13}=$ $W_{21}+W_{22}+W_{23}=W_{31}+W_{32}+W_{33}=1$
Then,

$$
\begin{aligned}
& {f^{\prime}{ }_{i 1}}=f_{i 1} W_{11}+f_{i 2} W_{21}+f_{i 3} W_{31} \\
& f^{\prime}{ }_{j 1}=f_{j 1} W_{11}+f_{j 2} W_{21}+f_{j 3} W_{31}
\end{aligned}
$$

We assume that $f_{i 1}>f_{j 1}$ and $f_{i 3}=f_{j 3}$, then $f^{\prime}{ }_{i 1}>f^{\prime}{ }_{j 1}$ under the condition

$$
\left(f_{i 1}-f_{j 1}\right) /\left(f_{j 2}-f_{i 2}\right)>W_{21} / W_{11}
$$

If we can assume that

$$
\begin{aligned}
& \quad W_{12}=W_{21}=W_{13}=W_{31}=W_{23}=W_{32}=W \\
& f_{i 1}^{\prime}>f_{j 1}^{\prime} \text { when }
\end{aligned}
$$

$$
W_{11}>W
$$

When $m$ equals three or more than three, the orders of items based on $f_{i 1} f_{i 2} f_{i_{3}}$ and so forth do not necessary coincide. If we need a combined order, there occurs the problem how to combine them. The problem remains for the future discussion.

\section{Reliability matrix}

One of the most serious defects in the present day scaling based on frequencies can be found in the fact that the reliability matrix is not known almost all cases. The same defects can be found in any experiments or research based on counting the number of responses. It is highly probable that the obtained data are biased owing to the imperfect reliability of the methods or the subjects. Psychologists usually put to much importance on expressing reliability in terms of correlation coefficient or other similar coefficients. Therefore, it is necessary to discuss the relationship between reliability matrix and correlation coefficient. We will restrict our discussion to the case where the reliability matrix is given in a form

$$
\left(\begin{array}{ll}
n W_{11} & n W_{12} \\
n W_{21} & n W_{22}
\end{array}\right)
$$

where $n$ is a constant related to the number of replication. It is obvious that $n W_{11}+$ $n W_{12}=n W_{21}+n W_{22}=n$. If we can calculate the phi coefficient from the matrix, it takes the form 


$$
\begin{aligned}
n^{2}( & \left.W_{11} W_{22}-W_{12} W_{21}\right) / n^{2} \\
& {\left[\left(W_{11}+W_{21}\right)\left(W_{12}+W_{22}\right)\right]^{1 / 2} } \\
= & \left(W_{11}-W_{21}\right) /\left[\left(W_{11}+W_{21}\right)\right. \\
& \left.\left(W_{12}+W_{22}\right)\right]^{1 / 2}
\end{aligned}
$$

It is obvious that $\varphi$ is positive when $W_{11}$ $>W_{21}$. This means that the condition (22) holds when $\varphi$ is positive. If we calculate from $B$ in (19) the necessary cross product, it takes the form

$$
\left(W_{11}-W_{21}\right)\left(f_{i 1} f_{j 2}-f_{j 1} f_{i 2}\right)
$$

Therefore, $\varphi$ takes positive value when $W_{11}$ $>W_{21}$ and $f_{i 1} f_{j 2}>f_{j 1} f_{i 2}$ or $W_{11}<W_{12}$ and $f_{i 1} f_{j 2}<f_{j 1} f_{i 2}$. If we could find a pair of items of which $f_{i 1} f_{2 j}>f_{1 j} f_{2 i}$ and if the obtained value of $\varphi^{\prime}$ is positive, we can conclude that the obtained order coincides with the original one.

Or if we can assume that $W_{11}=W_{22}$ and if we know the value of $\varphi$ calculated by (27), we can easily reproduce the reliability matrix. As $W_{11}+W_{21}=W_{11}+W_{12}=1$ and $W_{12}+W_{22}=W_{21}+W_{22}=1$,

$$
W_{11}-W_{21}=W_{11}-W_{12}
$$

For example, if $\varphi=0.80$

$$
\begin{aligned}
& W_{11}-W_{12}=0.80 \text { and } W_{11}+W_{12}=1.00 \\
& W_{11}=0.90 \text { and } W_{12}=0.10
\end{aligned}
$$

But the problem is to be left for the future discussion.

\section{Concluding remarks}

The present discussion does not cover all of the important problems concerning the application of the principle of frequency distribution. We have omitted the case of the pick $m$ method where subject is requested to choose $m$ objects out of $n$. We have also omitted the case of multi-dimensional ordinal scaling and have restricted our main concern to the case of unidemensional scales.

Psychologists tend to think the error factors in terms of sampling statistics when dealing with frequency data although the classical psychophysics already pointed out the possibility of many kinds of constant errors. We should not ignore the fact that we are dealing with biased data or possibly biased ones when we deal with frequency data.

Latent structure analysis proposed by Lazarsfeld $(1959,1960)$ is based on the similar idea but the theory deals with much more sophisticated problems. Ability scale proposed by Lord deals with the similar notion (1952, 1953, 1959, 1960), but unfortunately it takes an assumption that ". . . errors of measurement is that they are chance variables, each with a mean value that is always zero, irrespective of the value of the true score, or of the value of any other error of measurement." (1960, p. 101).

We do not deny that there may be many cases where such an assumption may hold true. What we want to suggest is that there are many cases where such an assumption does not hold.

\section{REFERENCES}

LAZARsFeld, P.F. 1959 Latent structure analysis. In S. Koch (Ed.), Psychology. Vol. III. New York: McGraw-Hill.

LAZARSFELD, P. F. 1960 Latent structure analysis and test theory. In $\mathrm{H}$. Gulliksen \& $\mathrm{S}$. Messick (Eds.), Psychological scaling. New York: John Wiley.

LORD, F. M. 1952 A theory of test score. Psychometric Monogr., No. 7.

LoRD, F. M. 1953 An application of confidence intervals and of maximum likelihood to the estimation of an examinee's ability. Psychometrika, 18, 57-76.

LoRD, F. M. 1959 Statistical inferences about true scores. Psychometrika, 24, 1-17.

LORD, F. M. 1960 Inferring the examinee's score. In H. Gulliksen \& S. Messick (Eds.), Psychological scaling. New York: John Wiley.

TanakA, Y. 1966 Some basic problems of the measurement in nominal scale. Jap. psychol. Research. 8, 53-61.

(Received July 1, 1966) 